\title{
Comparison of lung deposition of colomycin using the HaloLite and the Pari LC Plus nebulisers in patients with cystic fibrosis
}

\author{
N M Byrne, P M Keavey, J D Perry, F K Gould, D A Spencer
}

See end of article for authors' affiliations

Correspondence to Mrs N Byrne,

Rehabilitation Department, Royal Victoria Infirmary,

Queen Victoria Road,

Newcastle upon Tyne

NEl 4LP, UK ;

nuala.byrne@

nuth.northy.nhs.uk

Accepted

4 November 2002
Aim: To examine the effectiveness of delivery of nebulised colistin by the HaloLite nebuliser compared to the Pari LC Plus in patients with cystic fibrosis.

Methods: Randomised crossover trial of 15 patients aged $>6$ years. Inhalation of one mega unit of colistin in $3 \mathrm{ml}$ diluent, labelled with technetium-99m DTPA, was used to assess lung deposition. The Pari was nebulised to dryness and one button press of the HaloLite was completed. Following a seven day washout period, patients inhaled colistin twice daily for seven days through the first device. Sputum specimens were analysed for colistin levels and pseudomonas load. This procedure was repeated with the alternative device.

Results: Lung uptake of radiolabelled colistin was significantly higher with the Pari. However, lung uptake calculated as a percentage of the amount of drug used was significantly higher for the HaloLite. Time to nebulise was significantly shorter with the HaloLite. Sputum levels of colistin were higher following use of the Pari; this was close to significance.

Conclusion: The manufacturer's recommended dosages for nebulising antibiotics with a HaloLite result in a lower delivery than patients receive when using a Pari nebuliser. The concept of adaptive aerosol delivery has several theoretical advantages but the recommended doses for the Halolite need to be modified in order to improve effectiveness.
$\mathrm{P}$ rogressive lung disease in patients with cystic fibrosis (CF) is characterised by colonisation with Pseudomonas aeruginosa. ${ }^{1}$ Studies have shown that twice daily inhalation of antibiotics improves respiratory function, decreases hospital admissions, and reduces the frequency of positive pseudomonas cultures in recently colonised patients. ${ }^{2-4}$ The use of inhaled antibiotics is one of the few treatments shown by systematic review to slow down the decline in lung function and therefore increase longevity in patients with $\mathrm{CF}^{5}{ }^{6}$

Currently, administration of nebulised antibiotics is usually by use of breath enhanced nebuliser systems such as the Pari LC Plus (Pari Medical Ltd, Surrey, UK). There are multiple difficulties with this method, including unpredictability and variability of drug dosage, loss of drug substance to the environment especially during expiration, lengthy treatment times, no adaptation to the respiratory cycle of the patient, and variable adherence to treatment. ${ }^{78}$

The HaloLite nebuliser (Medic Aid Ltd, Bognor Regis, UK) is an adaptive aerosol delivery system which aims to overcome these problems. It analyses the pressure changes relating to airflow during the first three breaths, establishing the shape of the respiratory cycle and thereby ascertaining the correct starting point for aerosol delivery. It then delivers a pulse of aerosol during the first $50 \%$ of each inspiration and only pulses aerosol when the patient is inspiring through the mouthpiece. It is programmed to release a preset dose and signals audibly when the dose is complete. Proposed advantages of the system include more efficient delivery to the lungs and reduced loss of aerosol into the atmosphere. As completion of drug delivery is signalled by a bleep and time taken to nebulise could be less, adherence may be improved.

There are currently no published data examining the effectiveness of the HaloLite. Initial abstracts have indicated some improvement in adherence to treatment in asthmatic patients. ${ }^{90}$ In terms of drug delivery, it has been shown that in vitro, $80 \%$ of the particles were less than $5 \mu \mathrm{m}$ in diameter and there was a linear output to pulse time. ${ }^{11}$ The nebuliser has been tested in vivo in ten asthmatic and nine healthy subjects by assessing inhalation of saline and Tc-DTPA, showing that the HaloLite can accurately deliver a preset dose to the patient with less than $3 \%$ exhaled to the environment. ${ }^{11}$

None of this preliminary work included comparisons with existing nebuliser systems or investigation of antibiotic delivery in patients with CF. We have compared drug deposition achieved by the HaloLite and Pari LC Plus in children and young adults with CF.

\section{METHODOLOGY}

Fifteen subjects were recruited from the CF clinic at the Royal Victoria Infirmary, Newcastle upon Tyne. This number was based on a power calculation expecting a $15 \%$ reduction in the drug volume required by the HaloLite to achieve the same level of deposition in the lungs as the Pari. The power was $90 \%$ and the significance level was set at 0.05 . The regional ethics committee granted ethical approval. Inclusion criteria were a diagnosis of CF confirmed by two positive sweat tests and/or positive genotype, colonisation with Pseudomonas aeruginosa requiring daily inhalation of colistin (Colomycin, Forest Laboratories Europe), ability to expectorate sputum, aged $\geqslant 6$ years, and stable disease state. Patients who were colonised with Burkholderia cepacia, or had haemoptysis or pneumothorax were excluded from the study. Eligible patients were enrolled and randomly allocated as to which nebuliser they used first.

Following recruitment, each subject was provided with a HaloLite nebuliser system and taught how to use it correctly. Their technique with the Pari LC Plus and Pariboy compressor was also reviewed. Baseline measurements (age, height, weight, FVC, $\mathrm{FEV}_{1}$, and oxygen saturations) were recorded.

The study was divided into two sections. Lung deposition was measured firstly by inhalation of radiolabelled colistin 
Table 1 Baseline characteristics for all subjects participating in the study

\begin{tabular}{lllllll}
\hline & Age $(\mathrm{y})$ & Height $(\mathrm{cm})$ & Weight $(\mathrm{kg})$ & $\mathrm{FVC}(\%$ pred.) & $\mathrm{FEV}_{1}(\%$ pred.) & $\mathrm{SaO}_{2}(\%)$ \\
\hline Mean & 14.1 & 154 & 45.2 & 66.9 & 56.5 & 97 \\
$\mathrm{SD}$ & 4.6 & 15.8 & 11.7 & 19.5 & 18.9 & 1.7 \\
\hline
\end{tabular}

Table 2 Dosage used and lung uptake following inhalation of a dose of radiolabelled colistin using the Halolite and Pari nebulisers

\begin{tabular}{|c|c|c|c|c|c|c|}
\hline & \multicolumn{2}{|c|}{ Dosage used (ml) } & \multicolumn{2}{|c|}{ Lung uptake $(\mathrm{MBq})$} & \multicolumn{2}{|c|}{ Lung uptake (as \% dose used) } \\
\hline & HaloLite & Pari & HaloLite & Pari & HaloLite & Pari \\
\hline $\begin{array}{l}\text { Mean (SD) } \\
\mathrm{p} \text { value } \\
95 \% \mathrm{Cl}\end{array}$ & \multicolumn{2}{|c|}{$\begin{array}{c}<0.0001 \\
-2 \text { to }-1.47\end{array}$} & \multicolumn{2}{|c|}{$\begin{array}{c}<0.0001 \\
-8.1 \text { to }-3.98\end{array}$} & \multicolumn{2}{|c|}{$\begin{array}{c}<0.04 \\
-0.46 \text { to } 21.63\end{array}$} \\
\hline
\end{tabular}

solution and secondly by microbiological analysis of colistin levels and semiquantitative culture of pseudomonas load in sputum. Each subject participated in both arms of the study in random order. Subjects attended the medical physics department on two occasions, at least one week apart. Lung function was measured pre- and post-inhalation using a Vitalograph micro (Vitalograph) and subjects then inhaled their prescribed dose of $\beta$ agonist. In order to assess lung deposition, the nebuliser was primed with $60 \mathrm{MBq}$ Tc99m-DTPA and 1

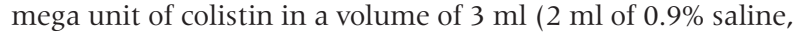
$1 \mathrm{ml}$ of water). The nebulisers were weighed before the priming dose was added and on completion of the nebuliser dose in order that residual drug volume could be calculated. The subjects were seated with their back resting against a Camstar XR/T (GE Medical Systems) gamma camera. The shielded nebuliser was placed on a table in front of the subject. During inhalation a dynamic acquisition of the posterior lungs was performed with a frame duration of 10 seconds and continuing for approximately one minute following the end of nebulisation. The end point for the Pari was dryness. The HaloLite stopped automatically when the preset dose had been delivered. One button press was completed for the HaloLite as recommended by the manufacturer. Immediately after inhalation, a posterior static view of the lungs was acquired with a total of 100000 counts. On completion, the subjects were asked to drink a glass of water to help remove any radioactive material from the oropharynx. At the next visit, the procedure was repeated using the second nebuliser.

For the microbiology section of the study, there was an initial washout period of seven days prior to usage of each nebuliser system. Following this, the subjects inhaled nebulised colistin twice daily from the first device for seven days. Sputum specimens were collected at one and four hours after the final dose. Following a further washout period of seven days, the procedure was repeated with the alternative device.

\section{Analysis of data}

Radionuclide data

The fraction of the priming dose used was calculated to give the nebulised activity in megabecquerels (MBq). Regions of interest were drawn around both lungs on the static images and the total lung counts per second were calculated after correcting for background activity using a C-shaped region of interest lateral to each lung. A conversion factor obtained from data on 18 patients undergoing standard lung perfusion scans was used to convert the lung counts to activity in MBq. Small differences in the priming dose added to the nebuliser were accounted for by normalising the data to a standard priming dose of $20 \mathrm{MBq} / \mathrm{ml}$. Lung activity was also expressed as a percentage of the dose which was actually nebulised.

Time-activity curves were produced from the dynamic acquisition after applying background correction and normalisation as described above. The time from the start of the acquisition to the beginning of a "plateau" region defined the duration of inhalation. A straight line was fitted to the inhalation phase and used to calculate the rate of uptake into the lungs.

For each patient, a common lung outline was produced from the HaloLite and Pari static images (the larger of the two) for each lung. This was used to define central and peripheral regions as described previously. ${ }^{12}$ The central region was a rectangle over the middle third of the medial border of

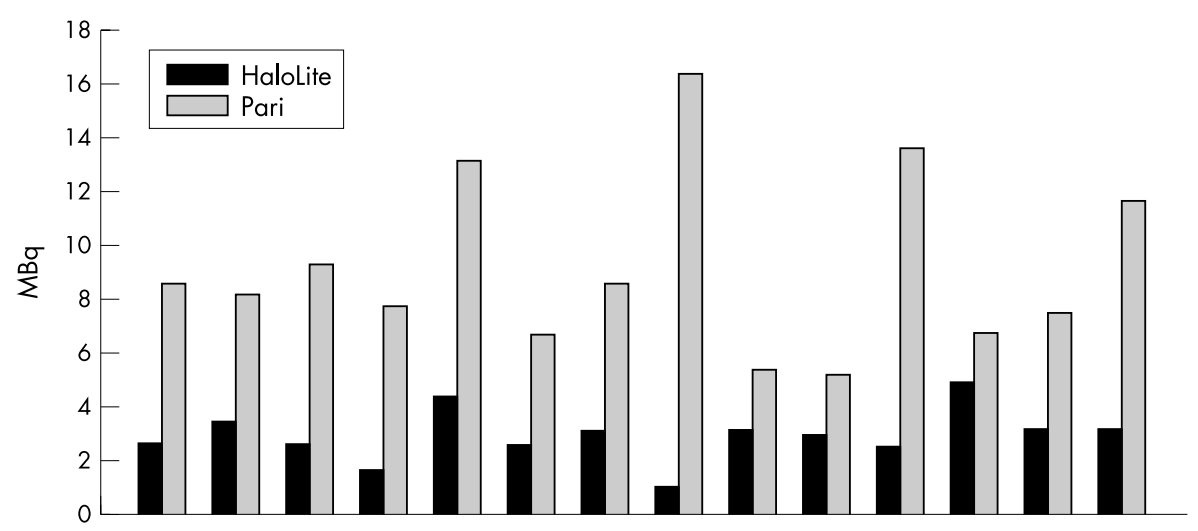

Figure 1 Lung deposition of radiolabelled colistin for each subject following use of the HaloLite and Pari LC Plus nebulisers.

Subjects 
Table 3 Mean differences for rate of aerosol uptake and levels of peripheral deposition using the HaloLite and Pari LC Plus nebulisers

\begin{tabular}{lll}
\hline & $\begin{array}{l}\text { Rate of aerosol uptake } \\
\text { (counts/second) }\end{array}$ & $\begin{array}{l}\text { Peripheral deposition } \\
(\%)\end{array}$ \\
\hline $\begin{array}{l}\text { Mean } \\
\text { difference }\end{array}$ & 9.62 & 1.34 \\
Standard error & 1.9 & 1.14 \\
\hline
\end{tabular}

the lung and extending halfway across the lung. The peripheral region was the remainder of the lung. A single C-shaped region of interest for each lung was used to estimate background activity for both the peripheral and central regions. An indication of the pattern of aerosol deposition was given by the uptake in the peripheral region expressed as a percentage of the total counts in the lung.

\section{Microbiology data}

Ideally, all sputum specimens were analysed on the day of collection. If this was not possible, specimens were refrigerated at a temperature of $0-4^{\circ} \mathrm{C}$ and tested within a maximum of 24 hours. Colistin levels were calculated by bioassay based on the procedure described by Grove and Randall (1955) and modified by the antibiotic reference laboratory at Southmead Hospital, Bristol, UK. A measured amount of sterile sputolysin sufficient for digestion was added to the sputum samples and left for 20-30 minutes. The quantity of liquid sputum was measured and the dilution factor calculated. This solution was then placed in wells made in agar plates and left to incubate overnight at $37^{\circ}$. All zone sizes produced were read the following day and concentrations in the samples were read from a standard curve and multiplied by the dilution factor.

Paired $t$ tests were used to analyse both the radionuclide data and the log normal distributed microbiology data.

\section{RESULTS}

Fifteen subjects aged 7-23 years participated in the study; 14 completed the radionuclide section and 13 the microbiology section. One subject became too ill and the second did not wish to complete the study. Table 1 presents baseline values for the whole sample.

There was no significant difference between the volume of the priming doses for each of the two nebuliser systems: mean (SD) $2.97(0.2) \mathrm{ml}$ for the HaloLite and $3.02(0.2) \mathrm{ml}$ for the Pari $(p=0.4)$. The HaloLite used significantly less drug volume than the Pari during each study (see table 2 ).

Lung uptake was significantly higher following use of the Pari nebuliser than the HaloLite. However, analysis of lung uptake as a percentage of the dose used showed that a greater percentage of the solution from the HaloLite than from the Pari was deposited in the lungs (see table 2 and fig 1 ).

Time to nebulise was less with the HaloLite (298 (50.14) seconds) than with the Pari $(382.5$ (68.3) seconds) $(\mathrm{p}<0.001,95 \%$ CI -133.5 to -41.49$)$. Rate of aerosol uptake was linear for both nebuliser systems but was significantly higher with the Pari $(\mathrm{p}<0.0006)$. There was no significant difference in percentage peripheral deposition of colistin between the two nebulisers $(\mathrm{p}=0.17)$. Table 3 shows mean differences (SE) for aerosol uptake and peripheral deposition.

Lung function results pre- and post-use of each nebuliser showed no significant change in $\mathrm{FEV}_{1}$ following use of either of the nebulisers (Pari, $\mathrm{p}=0.2$; HaloLite, $\mathrm{p}=0.6$ ).

In terms of microbiology results, drug delivery was log normally distributed as were levels of pseudomonas load. Colistin levels in sputum were higher following use of the Pari. These results were near to significance at one hour and not significant at four hours. Figure 2 illustrates values at one hour.

$P$ aeruginosa was not recovered from five specimens from the HaloLite group and six specimens from the Pari group. Table 4 presents results of sputum analysis.

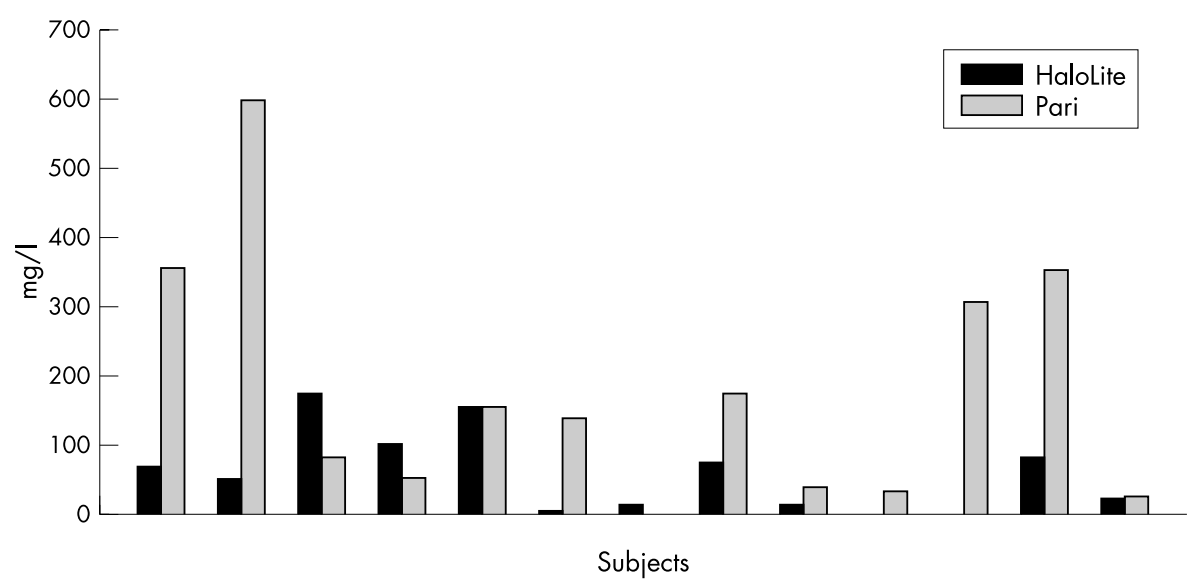

Figure 2 Colistin levels in sputum at one hour after use of the HaloLite and Pari LC Plus nebulisers.

Table 4 Geometric mean values, significance levels, and confidence intervals for colistin levels and pseudomonas load following use of the HaloLite and Pari nebulisers

\begin{tabular}{|c|c|c|c|c|}
\hline & \multicolumn{2}{|c|}{ Colistin levels (mg/l) } & \multicolumn{2}{|c|}{ Pseudomonas load (pseud/ml) } \\
\hline & 1 hour & 4 hours & 1 hour & 4 hours \\
\hline HaloLite & 64.2 & 51.8 & $1.2 \times 10^{6}$ & $3.7 \times 10^{6}$ \\
\hline Pari & 183.6 & 22.6 & $2.4 \times 10^{6}$ & $1.6 \times 10^{6}$ \\
\hline$p$ value & 0.06 & 0.6 & 0.9 & 0.5 \\
\hline $95 \% \mathrm{Cl}$ & -2.09 to 0.03 & -1.05 to 1.7 & -3.18 to 3.53 & -1.75 to 3.37 \\
\hline
\end{tabular}




\section{DISCUSSION}

The results of this study show that there is greater lung deposition of radiolabelled colistin solution using the Pari LC Plus system than with the HaloLite when using the manufacturer's recommended doses. However, percentage deposition of emitted drug solution was greater with the HaloLite, illustrating improved efficiency. Devadason and colleagues ${ }^{13}$ illustrated that there is a linear relation between one and two actuations of the HaloLite. Therefore, using the current preset doses, patients would need to carry out three button presses with the HaloLite to achieve the same level of deposition as with the Pari. Although time to nebulise was significantly less with the HaloLite in this study, this will be negated in clinical practice by the increased time needed for extra button presses.

These results are comparable with the results of a study by Devadason et al in children with CF. ${ }^{13}$ Delivery of rhDNase was measured using the HaloLite and the Pari LC Plus. Results showed that the Pari LC Plus was more effective and that at least two button presses of the HaloLite were needed to achieve an equivalent level of deposition. They also found a significantly higher level of peripheral deposition of radiolabelled DNase using the HaloLite in children with CF. In this study, a small difference was shown, with the HaloLite showing slightly greater peripheral deposition. However, this did not reach significance. The mass median diameter of particles emitted from the HaloLite $(3 \mu \mathrm{m})$ is smaller than those from the Pari $(3.8 \mu \mathrm{m})$. Theoretically, the HaloLite should therefore improve peripheral deposition. It may be that the method used here was not sufficiently sensitive to detect the difference. Separate studies using xenon or krypton gas would give a more accurate lung outline and allow better definition of the peripheral region. There have been reports that colistin can cause bronchoconstriction in patients with CF. ${ }^{14}$ Lung function results illustrated that no bronchoconstriction occurred following inhalation of colistin with either nebuliser.

A recognised difficulty with any nebuliser study carried out in a laboratory setting is that the subjects' adherence is likely to be far better than in "real life". Adherence is known to be generally poor in this group of patients. The HaloLite has been purported to improve adherence to treatment, but there is as yet no published data to substantiate this claim.

The microbiology data for pseudomonas load illustrated no significant difference between the two nebuliser systems. However, as five to six specimens from each group did not grow pseudomonas, it is likely that this represents a type two error. Colistin levels in sputum were higher when using the Pari compared to the HaloLite, and the difference was nearing significance. The difference between the two nebulisers in the microbiological assay was not as great as that found in the radionuclide section. There was a larger variability in individual data as can be seen in fig 2 , indicating that this was probably a less reliable method of measurement than the radionuclide data.

Commercially, a HaloLite nebuliser is five times more expensive than a Pariboy compressor and Pari LC Plus nebuliser. The HaloLite does use less colistin which is advantageous financially, but because of the extra button presses required, this may not be of economic advantage.

The concept of adaptive aerosol delivery is innovative in terms of improved patient technique and a reduction in the amount of colistin used. It may also have an effect on improving adherence to treatment. The current problem with the HaloLite is that the preset doses were originally based on inhaled budesonide and these do not correspond with the required dose of colistin. To achieve equivalent levels of colistin, patients would need to complete three button presses with the HaloLite. A possible alternative could be to increase the concentration of the colistin solution. This would require further work to ascertain the correct concentration. Very careful monitoring would be necessary as colistin is known to increase bronchospasm in some CF patients, ${ }^{15}{ }^{16}$ and this effect may be exacerbated by increasing the concentration.

Currently, nebuliser devices are not licenced, allowing new systems to be marketed with little or no data to prove their efficacy. The results of this study emphasise the need to move towards licensing of devices via the Medicines and Healthcare Products Regulatory Agency (formerly Medical Devices Agency). It also emphasises the need for objective assessment of individual devices with specific drugs used in patients with CF prior to their use in clinical practise.

\section{ACKNOWLEDGEMENTS}

We would like to thank Terry Hawkins for his intellectual contribution to the research. Technical support was given by Victoria Snowdon, for which we are very grateful. We would also like to thank the patients and their families for their participation. This research received support from Medic Aid Ltd who supplied the HaloLite nebuliser units.

........................

Authors' affiliations

N M Byrne, D A Spencer, Regional Paediatric Cystic Fibrosis Unit, Newcastle upon Tyne Hospitals NHS Trust, UK

P M Keavey, Regional Medical Physics Department, Newcastle upon Tyne Hospitals NHS Trust

J D Perry, F K Gould, Microbiology Department, Newcastle upon Tyne Hospitals NHS Trust

\section{REFERENCES}

1 Pitcher-Wilmott RW, Levinsky RJ, Gordon I. Pseudomonas infections, allergy, and cystic fibrosis. Arch Dis Child 1982;57:582-6.

2 Hodson ME, Penketh ARL, Batten JC. Aerosol carbenicillen and gentamycin treatment of Pseudomonas aeruginosa infection in patients with cystic fibrosis. Lancet 1981;ii: 1137-9.

3 Littlewood JM, Miller MG, Ghonheim AT, et al. Nebulised Colomycin for early pseudomonas colonisation in cystic fibrosis [letter]. Lancet 1985; 1:865

4 Valerius NH, Koch C, Hoiby N. Prevention of chronic Pseudomonas aeruginosa colonisation in cystic fibrosis by early treatment. Lancet 1999;338:725-6.

5 Touw DJ, Brimicombe RW, Hodson ME, et al. Inhalation of antibiotics in cystic fibrosis. Eur Respir J 1995;8:1594-604.

6 Mukhopadhyay S, Singh M, Cater Jl, et al. Nebulised anti-pseudomonal antibiotic therapy in cystic fibrosis: a meta analysis of benefits and risks. Thorax 1996:51:364-8.

7 Abbott J, Dodd M, Bilton D, et al. Treatment compliance in adults in cystic fibrosis. Thorax 1994;49:1 15-20.

8 Conway SP, Pond MN, Hamnett T, et al. Compliance with treatment in adult patients with cystic fibrosis. Thorax 1996;51:29-33.

9 Dyche JA, Medic Aid Ltd, Bognor Regis, UK. Patient compliance with inhaled treatment using a novel aerosol delivery system [abstract]. ERS Congress, Geneva. Eur Respir J 1998;(suppl):94s.

10 Nikander K, Denyer J, Cobos C. Compliance with nebulised budesonide in Spanish children with asthma using adaptive aerosol delivery (AAD) [abstract]. ERS Congress, Geneva. Eur Respir J 1998;(suppl):88s.

11 Denyer J, Dyche T, Nikander K, et al. HaloLite a novel liquid aerosol delivery system. Thorax 1997;52(suppl 6):A83.

12 O'Doherty MJ, Thomas SHL, Gibb D, et al. Lung deposition of nebulised pentamidine in children. Thorax 1993;48:220-6.

13 Devadason SG, Huang T, Walker SL, et al. Lung deposition of rhDNase in children with cystic fibrosis using the HaloLite Adaptive Aerosol Delivery $\left(A A D^{T M}\right)$ System and Pari $L C+$ nebuliser. European Cystic Fibrosis Conference, Vienna 2001, Abstract P84.

14 Chua HL, Collis GG, Le Souef PN. Bronchial response to nebulised antibiotics in children with cystic fibrosis. Eur Respir J 1990;3:11114-16.

15 Dodd ME, Abbott J, Maddison J, et al. Effect of tonicity of nebulised colistin on chest tightness and pulmonary function in adults with cystic fibrosis. Thorax 1997;52:656-8.

16 Cunningham S, Prasad A, Collyer L, et al. Bronchoconstriction following nebulised colistin in cystic fibrosis. Arch Dis Child 2001;84:432-3. 